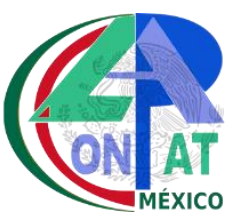

\title{
EFECTO DE LA ADICIÓN DE CBCA PROVENIENTE DE UN TRAPICHE INDUSTRIALIZADO DE LA CD DE IBAGUÉ, COLOMBIA. EN LA PROPIEDADES FÍSICAS Y MECÁNICAS DE UN SUELO TIPO AFIRMANTE
}

\author{
P. J. GALLEGO-QUINTANA ${ }^{1,2}$, A. ÁLVAREZ-ROSARIO², F. J. OLGUÍN-COCA ${ }^{3}$, \\ L. D. LÓPEZ LEÓN ${ }^{3}$, F. ALMERAYA ${ }^{4}$, O. F. OJEDA FARÍAS ${ }^{5}$, J. M. MENDOZA-RANGEL ${ }^{5}$, \\ M. A. BALTAZAR-ZAMORA ${ }^{6}$ \\ ${ }^{1}$ Universidad Veracruzana, Doctorado en Ingeniería, FIME, Xalapa, Veracruz, México \\ ${ }^{2}$ Universidad Cooperativa de Colombia, Facultad de Ingeniería Civil, Ibagué, Tolima, Colombia \\ ${ }^{3}$ Universidad Autónoma del Estado de Hidalgo, Mineral de la Reforma, Hidalgo. México. \\ ${ }^{4}$ Universidad Autónoma de Nuevo León. FIME - CIIIA. Av. Universidad S/N. \\ Ciudad Universitaria. San Nicolás de los Garza, Nuevo León, México \\ ${ }^{5}$ Universidad Autónoma de Nuevo León, FIC, San Nicolás de los Garza, N.L, México, \\ ${ }^{6}$ Universidad Veracruzana, Facultad de Ingeniería Civil - Xalapa, Circ. G. Aguirre Beltrán S/N, \\ Lomas del Estadio, CP 91000, Xalapa, Veracruz, México
}

\section{RESUMEN}

En el presente trabajo de investigación se evaluó el comportamiento de la Compactación y CBR (Ensayos de Limpieza y Resistencia del material) en base a las normas Colombianas INVIAS, para un suelo de tipo afirmante, con inclusión de 5, 10 y $15 \%$ de CBCA, residuo agroindustrial con características puzolánicas. A cada suelo se le realizaron los ensayos descritos con una repetitividad para los ensayos mecánicos de 4 especímenes. De acuerdo con los resultados de limpieza de material el suelo de estudio es un SW-SM de acuerdo con la clasificación del SUCS y un suelo A-1-a para la clasificación AASHTO. El valor de CBR se incrementa considerablemente con el aumento en la inclusión de la CBCA, llegando a más de un 77\% de incremento con el 5\% de CBCA, y de más del $300 \%$ con la inclusión del 10 y $15 \%$ de CBCA, lo que conlleva a que el suelo cumpla con los requerimientos de un material de Base.

Palabras clave: Compactación, CBR, CBCA, Suelo Afirmante.

\section{ABSTRACT}

In the present work of investigation, the behavior of the Compaction and CBR (Tests of Cleaning and Resistance of the material) was evaluated on the basis of the Colombian norms INVIAS, for a soil of affirming type, including 5, 10 and 15\% of CBCA, agroindustrial waste with pozzolanic characteristics. The described tests were performed on each soil with a repetitiveness for the mechanical tests of 4 specimens. According to the material cleaning results, the study floor is a SW-SM according to the classification of the SUCS and an A-1-a soil for the AASHTO classification. The value of CBR increases considerably with the increase in the inclusion of the CBCA, reaching more than $77 \%$ increase with $5 \%$ of CBCA, and more than $300 \%$ with the inclusion of 10 and $15 \%$ of CBCA, what leads to the soil meets the requirements of a base material.

Keywords: Compaction, CBR, SCBA, Affirming Soil. 


\section{RESUMO}

No presente trabalho de investigação o comportamento da Compactação e CBR (Testes de Limpeza e Resistência do material) foi avaliado com base nas normas colombianas INVIAS, para um solo de tipo afirmativo, incluindo 5, 10 e 15\% de CBCA, resíduo agroindustrial com características pozolânicas. Os testes descritos foram realizados em cada solo com uma repetitividade para os testes mecânicos de 4 exemplares. De acordo com os resultados de limpeza do material, o piso do estudo é um SW-SM de acordo com a classificação do SUCS e um solo A-1-a para a classificação AASHTO. O valor da RBC aumenta consideravelmente com o aumento da inclusão do CBCA, alcançando mais de $77 \%$ de aumento com 5\% do CBCA, e mais de 300\% com a inclusão de 10 e 15\% do CBCA, o que leva ao solo atende aos requisitos de um material de base.

Palavras-chave: Compactação, CBR, CBCA, Afirmando Solo

\section{INTRODUCCIÓN}

La experiencia demuestra que la construcción de carreteras conlleva a un deterioro de los ecosistemas, como lo plantea la federación europea de carreteras, definiendo a las carreteras sustentables como aquellas que son eficaces y eficientemente planeadas, diseñadas, construidas, modernizadas y conservadas, a través de políticas integradas con respecto al medio ambiente y conservan el beneficio socio-económico esperado en términos de movilidad y seguridad, y aunque hay literatura que indica estos puntos cruciales también se resalta que no se toman en cuenta el uso de materiales reciclados que pueden ser utilizados y que incrementarían aún más el aspecto sustentable de dichas carreteras (Mendoza, 2014).Ante la exposición de los aspectos previamente mencionados y el avance de la cultura en el reúso de los residuos de las diferentes industrias incluyendo las agroindustriales como la azucarera, en donde material de desecho proveniente de la combustión del bagazo de caña en las calderas de los Ingenios de Azúcar, conocido como Ceniza de Bagazo de Caña de Azúcar (CBCA), la cual no tiene ningún uso particular hasta la fecha, además de contaminar el ambiente (Escandon, 2012, Landa A.E. et.al., 2018; García D. et.al., 2019; Landa A. et.al.,2019), ha sido estudiado a nivel internacional debido a sus propiedades puzolánicas, demostrándose su gran potencial 'para ser utilizado como sustituto parcial del Cemento Portland (Chagas 2009, Cordeiro 2009), para la elaboración de concretos considerados por los investigadores como sustentables o ecológicos, evaluando sus propiedades mecánicas y aspectos de durabilidad como lo es la evaluación de la corrosión del acero de refuerzo cuando son expuestos a medios agresivos como cloruros y sulfatos (Landa A.E. et.al., 2018; Ruíz, M. 2019; Landa A.E. et.al., 2018; Santiago, G. 2016; Landa A. et.al.,2019) y en menor medida, pero más reciente en el área de estabilización y mejoramiento de suelos como los trabajos realizados en México (Ojeda O., 2015; Garcia D., 2017). Teniendo en cuenta que la industria cementera es la responsable de la emisión de del 7\% de las emisiones totales a nivel mundial de CO2 (Landa A.E. et.al., 2018; Ariza H. et.al., 2019; Baltazar M. et.al., 2016; Moraes et al., 2015 Santiago, G. 2016; Garcia D. et. al., 2019). De lo anterior el plantear la sustitución parcial del cemento portland por un material que en nuestro días al menos en Colombia es considerado desecho industrial, contribuiría a reutilizar los residuos de Ceniza de bagazo de Caña producidos por la quema de 24.380 .593 toneladas de caña procesada (Asocaña, 2018) y ser utilizadas completamente en las carreteras de Colombia como estabilizante haciendo más sustentables dichas carreteras, como lo indican algunos autores "la ciencia de la sostenibilidad puede fortalecer sus contribuciones empíricas, teóricas y prácticas, desarrollándose a lo largo de cuatro vías de investigación centradas en el papel de los valores de la ciencia y la toma de decisiones para la sostenibilidad" (Miller 2014). 


\section{PROCEDIMIENTO}

Determinar las propiedades físico-mecánicas del suelo granular antes y después de la adición de CBCA. El resultado de la evaluación de las características físicas y mecánicas del material granular virgen y estabilizado, son verificados con los requisitos de los agregados tipo afirmado definidos en la tabla 3111 y 311-2 del artículo 311 de las especificaciones para materiales para carreteras (INVIAS, 2013) con el fin de garantizar el cumplimiento del material para la construcción de carreteras.

\subsection{Materiales de estudio.}

El tipo de suelo analizado proviene de material de canto rodado del rio La Caima, el cual nace en la cordillera central colombiana, la cual hace parte de la cordillera de los Andes y el cual posee condiciones particulares que hacen que su comportamiento mecánico sea muy favorable para la construcción de carreteras., cemento portland compuesto 30R y ceniza de bagazo de caña de azúcar.

\subsection{Ensayos requeridos para el uso de suelo como afirmante normas INVIAS.}

Cada material o suelo que se considere utilizar en la construcción de carreteras se le deben realizar ensayos para su caracterización física y mecánica. El resultado de la evaluación de las características físicas y mecánicas del material granular virgen y estabilizado, son verificados con los requisitos de los agregados tipo afirmado definidos en la tabla 311-1 y 311-2 del artículo 311 de las especificaciones para materiales para carreteras (INVIAS, 2013) con el fin de garantizar el cumplimiento del material. Los ensayos realizados al suelo utilizado para el presente estudio fueron los de Limpieza (F), Límite Líquido norma E-125, Índice de plasticidad norma E-126, Contracción Lineal norma E-127, [Límites de Consistencia]. Ensayo de Resistencia del Material (F), CBR (\%): Porcentaje asociado al grado de compactación mínimo (numeral 311.5.2.2.2); el CBR se medirá sobre muestras sometidas previamente a cuatro días de inmersión, norma E-148.

\subsection{Cemento Portland como estabilizador de suelos.}

Se tiene de acuerdo a la literatura que el Cemento Portland es el material cementante más utilizado para la estabilización y mejoramiento de las propiedades mecánicas de los suelos en las obras civiles, más comúnmente en las construcciones de carreteras (Halsted, G., 2008). Se tiene que entre el 2 y el $25 \%$ de adición de cemento por peso de suelo es el rango más utilizado, pero el porcentaje de más de $15 \%$ se considera no económico. En la siguiente tabla se resume la recomendación de la AASHTO de adición de Cemento Portland de acuerdo con el tipo de suelo.

Tabla 1. Cemento requerido por volumen para la estabilización efectiva de varios suelos.

Tabla 1. Cemento requerido por volumen para la estabilización efectiva de varios suelos.

\begin{tabular}{|c|c|c|}
\hline \multicolumn{2}{|c|}{ Tipo de suelo } & \\
\hline $\begin{array}{c}\text { Clasificación } \\
\text { ASSHTO }\end{array}$ & SUCS & \% de Cemento \\
\hline A-2 Y A-3 & GP, SP Y SW & $6-10$ \\
\hline A-4 Y A-5 & CL, ML Y MH & $8-12$ \\
\hline A-6 Y A-7 & CL, CH & $12-14$ \\
\hline
\end{tabular}

\subsection{Porcentajes de ceniza y cemento utilizados.}

Para la evaluación del comportamiento físico y mecánico del suelo utilizado en el presente estudio, fue necesario la elaboración de 4 especímenes para la muestra control de material granular tipo afirmado y 4 especímenes por cada dosificación $(5 \%, 10 \%$ y $15 \%)$ de los diferentes porcentajes de inclusión de CBCA, el anterior procedimiento experimental se ejecutó con base en los documentos técnicos para 
carreteras del Instituto Nacional de Vías de Colombia, los cuales se tipifican en la sección 600, la tabla siguiente se resume el arreglo experimental.

Tabla 2. Arreglo experimental.

\begin{tabular}{|c|c|c|}
\hline Suelo Afirmado & $\%$ CBCA & S.A:CBCA \\
\hline $100 \%$ & $0 \%$ & $100: 0$ \\
\hline $100 \%$ & $5 \%$ & $100: 05$ \\
\hline $100 \%$ & $10 \%$ & $100: 10$ \\
\hline $100 \%$ & $15 \%$ & $100: 15$ \\
\hline
\end{tabular}

\section{RESULTADOS Y ANÁLISIS.}

\subsection{Límites de consistencia, clasificación AASHTO y SUCS.}

Al realizarse los ensayos para determinar los límites de consistencia, las muestras ensayadas de la zona de estudio, arrojaron valores particularmente bajos para los límites de consistencia (límite líquido y del límite plástico). Al realizar los ensayos con el suelo en su estado natural se obtuvieron valores de $\mathrm{LL}=$ $25.3 \%, \mathrm{LP}=21.6 \%$ y un $\mathrm{IP}=3.7 \%$.

\subsection{Compactación ASSHTO}

Con relación a la compactación, la cual se realizó acorde al procedimiento definido en la norma de ensayo denominada "relaciones humedad - peso unitario seco en los suelos (ensayo modificado de compactación) INV E - 142 - 13". A continuación la tabla 3 presenta los resultados del ensayo de compactación y en la figura 1 se observa la curva de compactación típica.

Tabla 3. Datos de ensayo modificado de compactación Proctor.

\begin{tabular}{|c|c|c|c|}
\hline \multicolumn{4}{|c|}{ DATOS DE LABORATORIO } \\
\hline Molde No. & 2 & 2 & 2 \\
\hline Peso Muestra Húmeda Compactada + Molde (g) & 7390.0 & 7556.0 & 7592.0 \\
\hline Peso Molde (g) & 2952.0 & 3045.0 & 3130.0 \\
\hline Peso Muestra Húmeda Compactada (g) & 4438.0 & 4511.0 & 4462.0 \\
\hline$\%$ de Humedad & $4.99 \%$ & $7.02 \%$ & $9.01 \%$ \\
\hline Peso Muestra Seca (g) & 4435.8 & 4507.8 & 4458.0 \\
\hline Volumen del Molde (g) & 2139.0 & 2139.0 & 2139.0 \\
\hline Densidad Húmeda $\left(\mathrm{g} / \mathrm{cm}^{3}\right)$ & 2.075 & 2.109 & 2.086 \\
\hline Densidad Seca de Laboratorio & 2.074 & 2.107 & 2.084 \\
\hline
\end{tabular}



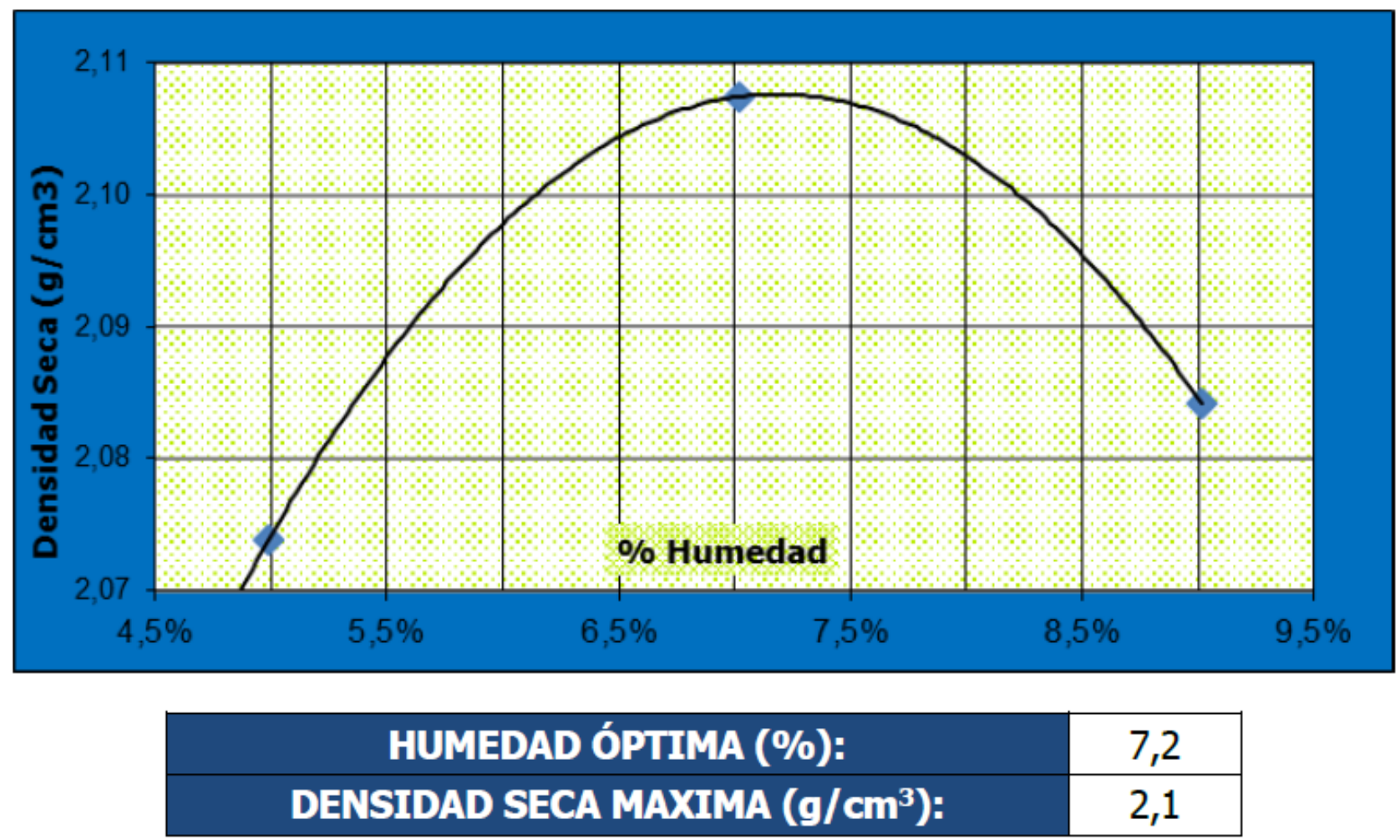

Figura 1. Gráfica de compactación.

\subsection{Ensayo de relación de Soporte del Suelo (CBR)}

Para la determinación de la resistencia mecánica de los materiales utilizados para la construcción de carreteras, es necesario realizar a los mismos el ensayo de relación de soporte del suelo en el laboratorio (CBR de laboratorio) I.N.V. E - 148 (INVIAS, 2007), los resultados obtenidos para en el documento técnico anteriormente referenciado, se describe el procedimiento para la elaboración y compactación de probetas con suelo granular y las diferentes proporciones de adición de CBCA.

En la tabla 3 se presentan los resultados obtenidos del ensayo CBR del material tipo afirmante sin adición de CBCA y con adición de 5, 10 y $15 \%$ de CBCA. Se ve incrementado su CBR el suelo afirmante en un $77 \%$ cuando la inclusión de un $5 \%$ de CBCA, para el caso del suelo con la inclusión de un 10 y $15 \%$ de CBCA, los valores de CBR se incrementan en más de tres veces el valor del suelo afirmante que fue de $35 \%$ a $5.08 \mathrm{~mm}$ de penetración. El incremento del CBR se asocia al efecto filler de la CBCA, en otros estudios en donde se estudió el efecto en el CBR de la de la adición de combinaciones de Cemento Portland-Ceniza de Bagazo de Caña de Azúcar en diferentes porcentajes también se observó un desempeño muy favorable con incrementos significativos en el CBR del suelo en estudio (Ojeda et. al., 2018). Los resultados de la presente investigación demuestran una mayor resistencia de la capa de afirmante, o suelo de estudio, lo que significaría que para el caso de Colombia, dicho suelo tipo afirmante presenta con la adición de CBCA, valores de CBR que cumplirían perfectamente con los requerimientos para ser utilizado para las capas de Sub-Base y Base. 
Tabla 4.- Datos de ensayo modificado de compactación Proctor.

AFIRMADO SIN CENIZA 0\%

AFIRMADO CON CENIZA 5\%

\begin{tabular}{|c|c|c|c|c|c|c|c|}
\hline \multicolumn{2}{|c|}{ PENETRACIÓN } & CARGA Ib/f & PRESIÓN PSI & CBR (\%) & CARGA Ib/f & PRESIÓN PSI & CBR (\%) \\
\hline $\mathrm{mm}$ & 0 & 0 & 0 & & 0 & 0 & \\
\hline 0,64 & 0,025 & 154 & 49,0197225 & & 154 & 49,0197225 & \\
\hline 1,27 & 0,05 & 309 & 98,3577548 & & 316 & 100,585924 & \\
\hline 1,91 & 0,075 & 455 & 144,830998 & & 551 & 175,388747 & \\
\hline 2,54 & 0,1 & 646 & 205,628186 & $\mathbf{2 1} \%$ & 874 & 278,202841 & $\mathbf{2 8} \%$ \\
\hline 3,18 & 0,125 & 867 & 275,974671 & & 1285 & 409,028204 & \\
\hline 3,81 & 0,15 & 1102 & 350,777495 & & 1675 & 533,169059 & \\
\hline 4,45 & 0,175 & 1374 & 437,357784 & & 2306 & 734,022598 & \\
\hline 5,08 & 0,2 & 1667 & 530,62258 & $35 \%$ & 2916 & 928,191628 & $\mathbf{6 2} \%$ \\
\hline 7,62 & 0,3 & 3225 & 1026,54938 & & 5612 & 1786,35508 & \\
\hline 9,52 & 0,375 & 4561 & 1451,81139 & & 7860 & 2501,91571 & \\
\hline
\end{tabular}

\begin{tabular}{|c|c|c|c|c|c|c|c|}
\cline { 2 - 8 } \multicolumn{1}{c|}{} & \multicolumn{3}{c|}{ AFIRMADO CON CENIZA 10\% } & \multicolumn{3}{c|}{ AFIRMADO CON CENIZA 15\% } \\
\hline \multicolumn{2}{|c|}{ PENETRACIÓN } & CARGA Ib/f & PRESIÓN PSI & CBR (\%) & CARGA Ib/f & PRESIÓN PSI & CBR (\%) \\
\hline mm & 0 & 0 & 0 & & 0 & 0 & \\
\hline 0,64 & 0,025 & 800,666667 & 254,860116 & & 1065 & 339,000029 & \\
\hline 1,27 & 0,05 & 2088,66667 & 664,843249 & & 3783 & 1204,1663 & \\
\hline 1,91 & 0,075 & 3462 & 1101,98883 & & 6511,5 & 2072,67482 & \\
\hline 2,54 & 0,1 & 4634,66667 & 1475,26022 & $148 \%$ & 8976,5 & 2857,30869 & $198 \%$ \\
\hline 3,18 & 0,125 & 5793 & 1843,96917 & & 10996 & 3500,13551 & \\
\hline 3,81 & 0,15 & 6941,66667 & 2209,60113 & & 12759 & 4061,31584 & \\
\hline 4,45 & 0,175 & 8070 & 2568,76078 & & 14382 & 4577,93278 & \\
\hline 5,08 & 0,2 & 9277,33333 & 2953,06692 & $197 \%$ & 15528 & 4942,71591 & $172 \%$ \\
\hline 7,62 & 0,3 & 12373,5 & 3938,60738 & & 20471,5 & 6516,28084 & \\
\hline 9,52 & 0,375 & 15359 & 4888,92154 & & 23314 & 7421,07669 & \\
\hline
\end{tabular}

\section{CONCLUSIONES.}

De acuerdo con los estudios realizados se puede concluir lo siguiente:

- Se registraron muy significativos incrementos los valores obtenidos del ensayo de CBR en un suelo tipo afirmante, clasificado como SW-SC de acuerdo con el SUCS y A-1-a de acuerdo con la AASHTO.

- El incremento con una inclusión de un 5\% de CBCA al suelo tipo afirmante incremento en un $77 \%$ el CBR.

- Al llevar la inclusión de CBCA en un $10 \%$ y $15 \%$ se triplico el valor de CBR del suelo en estudio. Los valores de CBR obtenidos cumplen con los requeridos para las capas de Base y Sub-Base de acuerdo con la norma de INVIAS.

- El incremento se asocia al efecto filler de la CBCA 


\section{AGRADECIMIENTOS}

M.A. Baltazar-Zamora, et. al., agradecen a PRODEP por el Apoyo otorgado por la SEP, al Cuerpo Académico UV-CA-458 "Sustentabilidad y Durabilidad de Materiales para la Infraestructura Civil", en el marco de la Convocatoria 2018 para Fortalecimiento de Cuerpos Académicos con IDCA 28593.

\section{REFERENCIAS}

Ariza H.A., Croche R., Gaona C., Almeraya F., Ramírez A.C., Villegas R., Márquez S., BaltazarZamora M.A. (2019). "Evaluación de la corrosión del acero galvanizado y acero AISI 304 embebidos enconcretos ecológicos con sustitución de CPC por 10, 20 y 30\% de CBCA-HS expuestos a Na2SO4 al 3.5\%". Memorias del XXXIV Congreso Nacional de la Sociedad Mexicana de Electroquímica, 12th Meeting of the Mexican Section of the Electrochemical Society, SMEQ, Querétaro (México).

ASOCAÑA (2018), Balance azucarero colombiano Asocaña 2000-2018. Disponible en: http://www.asocana.org/modules/documentos/3/194.aspx, consultado en 4/24/2018.

Baltazar-Zamora M.A., Santiago, G., Moreno V., Croche R., De la Garza M., Estupiñan, F., Zambrano, P., Gaona, C. (2016). Electrochemical Behaviour of Galvanized Steel Embedded in Concrete Exposed to Sand Contaminated with $\mathrm{NaCl}$. Int. J. Electrochem. Sci. 11, 10306-10319, doi: 10.20964/2016.12.28. Chagas C. G., Dias R. T. F., Marcelo L. T., Rego E.M.F. (2009). Ultrafine grinding of sugar cane bagasse ash for application as pozzolanic admixture in concrete. Cement and Concrete Research 39 (2009) 110-115

Cordeiro, G.C., Toledo, R.D., Tavares, E.R., Fairbairn E.M. (2008). Pozzolanic activity and filler effect of sugar cane bagasse ash in Portland cement and lime mortars. Cement and Concrete Composites. 30(5):410-418

Escandon G, C. E., Vidal Velasco, D. V., Martínez López, C., Torres Ägredo, J., \& González Salcedo, L. O. (2012). Ceniza de bagazo de caña como aditivo al cemento portland para la fabricación de elementos de construcción. Acta Agronómica, 77-78.

Frias, M., Villar, E., Savastano, H. (2011). Brazilian sugar cane bagasse ashes from the cogeneration industry as active pozzolans for cement manufacture. Cement and Concrete Composites, 490-499.

García García, D.L. (2017). Influencia de la ceniza de bagazo de caña como sustituto de cemento portland en la estabilización de un material para sub-base. Tesis de Licenciatura, Facultad de Ingeniería Civil, U.V., Xalapa, Veracruz, México.

García D.L, Cabral J.A, Gaona C., Almeraya F., Ramírez A.C., Croche R., Baltazar-Zamora M.A. (2019). "Influencia del medio ambiente de exposición y tipo de curado en la cinética de corrosión de concreto reforzado" Memorias del XXXIV Congreso Nacional de la Sociedad Mexicana de Electroquímica, 12th Meeting of the Mexican Section of the Electrochemical Society, SMEQ, Querétaro (México).

García D.L, Cabral J.A, Gaona C., Almeraya F., Ramírez A.C., Villegas R., Marquez S., BaltazarZamora M.A. (2019). "Eficiencia anticorrosiva del acero AISI 304 como refuerzo en concretos ecológicos ternarios base CBCA-HS expuestos a sulfato de magnesio". Memorias del XXXIV Congreso Nacional de la Sociedad Mexicana de Electroquímica, 12th Meeting of the Mexican Section of the Electrochemical Society, SMEQ, Querétaro (México).

Halsted, G., Adaska, W.S., McConnell, W.T. (2008). Guide to Cement-Modified Soil (CMS). Skokie, Illinois: Portland Cement Association.

I.N.V. (2013). E - 148 - 07. RELACIÓN DE SOPORTE DEL SUELO EN EL LABORATORIO (CBR DE LABORATORIO), COLOMBIA.

Jofre, C., Kraemer, C., Sampedro, A., Lopez Bachiller, A., Atienza, M., Diaz, M. (2008). Manual de estabilización de suelos con cemento o cal. Madrid: Instituto Español del cemento y sus aplicaciones. 
Landa A., Gaona C., Almeraya F., Ramírez A.C., López O. M., Vélez J.A., Solorzano R., Márquez S., Baltazar-Zamora M.A., (2019). "Evaluación electroquímica del acero inoxidable AISI 316 embebido en concretos sustentables base ceniza de bagazo de caña de azúcar expuestos a un medio marino" Memorias del XXXIV Congreso Nacional de la Sociedad Mexicana de Electroquímica, 12th Meeting of the Mexican Section of the Electrochemical Society, SMEQ, Querétaro (México).

Landa A., Landa A., Rangel J.M., Almeraya F., Croche B., Baltazar-Zamora M.A. (2019). "Comportamiento de la corrosión del acero AISI 316 como refuerzo en concretos ternarios sustentables con sustitución parcial de CPC en 10\%, 20\% y 30\% de CBCA-HS inmersos en agua de mar". Memorias del XXXIV Congreso Nacional de la Sociedad Mexicana de Electroquímica, 12th Meeting of the Mexican Section of the Electrochemical Society, SMEQ, Querétaro (México).

Landa A.E., Croche R., López O.M., Galván R., Cabral J.A., Gaona C., Almeraya F., Baltazar-Zamora M.A (2018). Corrosion Behavior of AISI 316 Stainless Steel As Reinforcement in Ternary Sustainable Concrete Based on SCBA-SF Exposed in Seawater, ECS Meeting Abstracts, MA2018-02 584-584

Landa A.E., Castillo S., Reyes J.A., Villegas R., Galván R., Cabral J.A., Gaona C., Almeraya F., Baltazar-Zamora M.A. (2018). Evaluation of the Corrosion of AISI 304 Stainless Steel Embedded in Sustainable Concrete with High Volumes of Scba-SF Exposed in Marine Environment, ECS Meeting abstracts, MA2018-02 587

Landa A.E., Croche R., Márquez S., Villegas R., Ariza H.A., Estupiñan F., Gaona C., Almeraya F., Baltazar-Zamora M.A (2018). Corrosion Behavior 304 and 316 Stainless Steel as Reinforcement in Sustainable Concrete Based on Sugar Cane Bagasse Ash Exposed to $\mathrm{Na}_{2} \mathrm{SO}_{4}$, ECS transaction, 84, 179188

Landa A.E., Croche R., Márquez S., Galván R., Gaona C., Almeraya F., Baltazar-Zamora M.A. (2018). Correlation of Compression Resistance and Rupture Module of a Concrete of Ratio $w / c=0.50$ with the Corrosion Potential, Electrical Resistivity and Ultrasonic Pulse Speed, ECS transactions, 84, 217-227 Mendoza Sánchez J. F. (2014). Criterios de sustentabilidad para carreteras en México. Publicación Técnica No. 392, IMT, Sanfandila, Qro.México. ISSN 0188-7297

Miller R., Wiek A., Sarewitz D., Robinson J., Olson L., Kriebel D., Loorbach D. (2014). The future of sustainability science: a research agenda oriented solutions 9: 239-246. Neuenschwander A. 2010. El Cambio Climático en el Sector Silvoagropecuario de Chile, fundación para la innovación agraria anexo 1 pp. 119

Moraes, J. C., Akasaki, J. L., Melges, J. L., Monzo, J., Borrachero, M. V., Soriano, L., et al. (2015). Assessment of sugar cane straw ash (SCSA) as pozzolanic material in blended portland cement: microstructural characterization of pastes and mechanical strength of mortars. Construction and Building Materials, 670-677.

Organismo Nacional de Normalización y Certificación de la Construcción y Edificación, S.C. (2014), "NMX-C-414-ONNCCE-2014: Industria de la Construcción - Cementantes Hidráulicos Especificaciones y Métodos de Ensayo", ONNCCE, S.C. México

Ojeda Farias, O. F. (2015), Influencia de la ceniza de bagazo de caña de azúcar como sustituto parcial del cemento portland en la estabilización de una arena, Tesis de Maestría, Facultad de Ingeniería Civil, U.V., Xalapa, Veracruz, México.

Ojeda Farías, O., Baltazar Zamora, M., \& Mendoza Rangel, J. (2018). Influence of sugar cane bagasse ash inclusion on compacting, CBR and unconfined compressive strength of a subgrade granular material. Revista ALCONPAT, 8(2), 194-208. http://dx.doi.org/10.21041/ra.v8i2.282

Ruíz M., Landa A., Cabral J.A., Gaona C., Almeraya F., Malpica R., Márquez S., Baltazar M.A. (2019), "Influencia del tipo de acero en el comportamiento de la corrosión de concretos sustentables ternarios elaborados con ceniza de bagazo de caña de azúcar y humo de sílice expuestos a sulfato de sodio" Memorias del XXXIV Congreso Nacional de la Sociedad Mexicana de Electroquímica, 12th Meeting of the Mexican Section of the Electrochemical Society, SMEQ, Querétaro (México). 
Santiago, G., Baltazar-Zamora M.A., Galván, R., López, L.D., Zapata, F., Zambrano, P., A. Gaona, C. and Almeraya, F. (2016), Electrochemical evaluation of reinforcement concrete exposed to soil type sp contaminated with sulphates. Int. J. Electrochem. Sci. 11,4850-4864, doi: 10.20964/2016.06.31 Santiago, G., Baltazar-Zamora M.A., Olguín, J., López, L.D., Galván, R., Ríos, A. Gaona, C. and Almeraya, F. (2016), "Electrochemical evaluation of a stainless steel as reinforcement in sustainable concrete exposed to chlorides". Int. J. Electrochem. Sci. 11, 2994 